\title{
Fano still resonating
}

\author{
More than half a century after describing interference of discrete states with a continuum, Ugo Fano's \\ work is as relevant as ever. And Fermi beat him to it.
}

The 'Fano effect' and its associated signature asymmetric spectrum ${ }^{1}$ will likely be familiar to many of our readers. The descriptions were general and today the concepts are still relevant to numerous modern photonics scenarios such as metamaterials and nanoantennae. In this issue, Mikhail Limonov and colleagues review the field of Fano resonances applied to current photonics ${ }^{2}$.

The life and scientific achievements of Ugo Fano (1912-2001) have been documented in several articles. A particularly informative essay $^{4}$ was penned by his first $\mathrm{PhD}$ student at the University of Chicago, Ravi Rau (now at Louisiana State University). If Fano were alive today, it would be interesting to ask whether he is surprised at the continued interest in the Fano effect. Rau says the answer is no. Other students of Fano concur.

Anthony Starace of the University of Nebraska, another former graduate student of Fano, told Nature Photonics that the fact "that different situations can be described by the same mathematical equations would not have been a surprise to Fano. He always stressed to his students that they begin attacking a problem by developing a completely general theoretical analysis before making approximations and simplifications applicable to particular situations."

Chris Greene of Purdue University (another former student of Fano) does, however, remember as a graduate student asking Fano in the late 1970s a question about his famous 1961 paper that revised and extended the theoretical explanation of the asymmetric peaks that appear in atomic excitation spectra. At that time, Fano seemed surprised people were still interested in that paper. "I remember clearly that he said the treatment of his 1961 paper was made 'obsolescent' by the development of multichannel quantum defect theory," Greene told Nature Photonics. Rau recalls a similar interaction. Fano told him that the 1961 formalism was obsolete and could be done much better, as in their 1986 book Atomic Collisions and Spectra ${ }^{5}$. The book, Rau explained, coupled the work with another of Fano's favourite themes; he liked to see spectroscopy (bound states) and collisions and scattering (continuum states) on an equal footing, belonging to the spectrum of the same Hamiltonian. While Greene does note the power of the later quantum

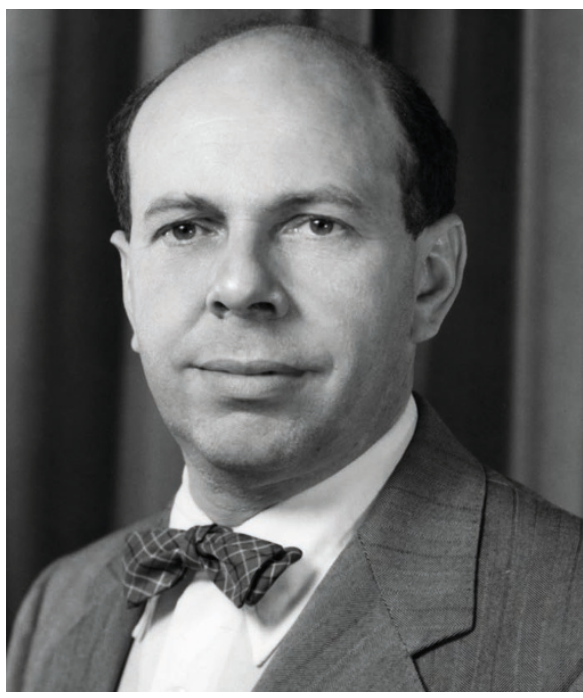

defect theory, he mentioned that the earlier 1961 Fano lineshape theory was arguably more broadly applicable, for example, to photonic systems and condensed-matter systems where quantum defect ideas are less obviously useful.

How did Fano come to work on spectra and their lineshapes? Starace explained that Fano was accepted into Enrico Fermi's group and was also taken under the wing of a young faculty member, Emilio Segre. "In those days, there was no salary," Starace explained. "Postdocs were content to have a desk and access to the professor they wished to work with." Starace and Greene told Nature Photonics that it was Fermi who suggested Fano come up with a theoretical description for the asymmetrical lineshapes of spectra of gases observed at that time in Fermi's laboratory.

Although he published the result in $1935^{6}$, Greene and Starace confirmed that some years later, Fano was browsing Fermi's notebooks and found that Fermi himself had solved the problem within days of presenting it to Fano. An account of the details, particularly well worth reading for any advisor of students, reveals the selfless and nurturing mentoring bestowed by Fermi ${ }^{4}$. Fermi may have solved the problem later the same day he gave it to Fano, but he refused co-authorship ${ }^{4}$.

It is important to note that Fano's contributions go well beyond these 'lineshape' works. With regard to his other important findings, both Starace and Greene mentioned the research on the description of states in quantum mechanics by density matrix and operator techniques ${ }^{7}$. Starace noted the particularly clear and physical presentation of the techniques applied to a variety of complex physical phenomena in that work and also paid tribute to another manuscript on spectral distribution of atomic oscillator strengths ${ }^{8}$, which set the stage for theoretical understanding of the new synchrotron atomic spectra in the vacuumultraviolet energy region.

While much has been written about Fano's achievements, less has been discussed about what kind of person he was to work with. Having arrived as a graduate student at the University of Chicago in 1966, Starace found Fano was personally very warm, demanding of his students and did not tolerate carelessness or foolishness. He was very interactive, joining the group for lunch at least three times a week, with discussions not limited to physics, but extending to politics and the arts. It was at these lunch meetings that Starace and the group saw another side of Fano, the passionate intellectual.

"Fano expected his students to get to the office before he did, typically after 9 am," commented Starace. "After getting settled, Fano would stroll into the two offices housing his graduate students and his postdoc, and address each one of us with the question, 'What's new?"' Fano would ask the same question in the late afternoon, and then again the next morning, which Greene recalled made it clear that they were supposed to have been working on their research projects during the evening hours.

Starace sums up by saying that life as a graduate student in Fano's group was great because of the intense interaction with Fano on a daily basis and that "one felt that one's work was important and one knew that Fano cared."

\footnotetext{
References

1. Fano, U. Phys. Rev. 124, 1866-1878 (1961).

2. Limonov, M. F., Rybin, M. V., Poddubny, A. N. \& Kivshar, Y. S. Nat. Photon. 11, 543-554 (2017).

. Clark, C. W. Nature 410, 164 (2001)

4. Rau, A. R. P. Phys. Essays 13, 158-175 (2001).

5. Fano, U. \& Rau, A. R. P. Atomic Collisions and Spectra (Academic Press, 1986).

6. Fano, U. Nuovo Cimento 12, 154-161 (1935). [English translation: Preprint at https://arxiv.org/abs/cond-mat/0502210; 2005.]

7. Fano, U. Rev. Mod. Phys. 29, 74-93 (1957).

8. Fano, U. \& Cooper, J. W. Rev. Mod. Phys. 40, 441-507 (1968).
} 